\title{
Statistical properties of type II Topp Leone inverse exponen- tial distribution
}

\section{Sanaa Al-Marzouki}

Statistics Department, Faculty of Science, King AbdulAziz University, Jeddah, Kingdom of Saudi Arabia.

\begin{abstract}
In this paper a new lifetime parameters model named the type II topp leone inverse exponential (TIITLIE) model is proposed. Some fundamental properties of the TIITLIE model are calculated. The maximum likelihood (ML) method of estimation are assessed to the parameters of TIITLIE distribution. The superiority of the TIITLIE model is showed by using 63 aircraft windshields real data set.
\end{abstract}

Keywords: Inverse exponential model, type II Topp Leone-G, moments, maximum likelihood.

2020 MSC: 60E05, 62E15, 62F10.

(C)2021 All rights reserved.

\section{Introduction}

The inverse exponential (IE) model is proposed by Keller and Kamath [2]. If $X$ is a non-negative random variable $(\mathrm{rv}), X \sim E$ model then the distribution of a $r v Y=1 / X$ follows an IE distribution. The pdf and cdf of the IE model, respectively, are

$$
g(x: \alpha)=\frac{\alpha}{x^{2}} e^{-\frac{\alpha}{x}}, \quad x, \alpha>0
$$

and

$$
G(x: \alpha)=e^{-\frac{\alpha}{x}}, \quad x, \alpha>0 .
$$

Recently some authors attempts to increase the generalization of the IE model. Singh and Goel [7] proposed Beta IE distribution, Kumaraswamy IE is introduced by Oguntunde et al. [3], transmuted IE distribution is investigated by Oguntunde et al. [4], Oguntunde et al. [5] studied exponentiated generalized IE model, Weibull-IE model is proposed by Oguntunde et al. [6].

Elgarhy et al. [1] introduced Type II Topp Leone generated (TIITL-G) distributions. The cdf and pdf of TIITL $-G$ are

$$
F(x: \theta, \xi)=1-\left[1-[G(x ; \xi)]^{2}\right]^{\theta}, \quad x \in R, \theta>0
$$

Email address: salmarzouki@kau.edu.sa (Sanaa Al-Marzouki)

doi: $10.22436 /$ jnsa.014.01.01

Received: 2019-01-07 Revised: 2019-01-20 Accepted: 2019-02-14 
and

$$
f(x: \theta, \xi)=2 \theta g(x ; \xi) \mathrm{G}(x ; \xi)\left[1-[\mathrm{G}(x ; \xi)]^{2}\right]^{\theta-1} .
$$

The rest of this article is arranged as follows. In Section 2, the TIITLIE model is proposed. Fundamental properties is calculated in Section 3. The ML method of estimation is perofrmed to show the behavior of the estimates of the TIITLIE parameters in Section 4. The application to one real data set is performed in Section 5. Finally, conclusions are mentioned in Section 6.

\section{The new model}

Let $\mathrm{rv} X \sim$ TIITLIE distribution, then, the cdf and pdf of TIITLIE model with parameters $\varphi=(\theta, \alpha)$ is obtained by inserting (1.1), (1.2) in (1.3) and (1.4) as

$$
F(x ; \varphi)=1-\left[1-e^{-\frac{2 \alpha}{x}}\right]^{\theta}, x, \theta, \alpha>0
$$

and

$$
f(x ; \varphi)=2 \theta \alpha x^{-2} e^{-\frac{2 \alpha}{x}}\left[1-e^{-\frac{2 \alpha}{x}}\right]^{\theta-1}, \quad x, \theta, \alpha>0
$$

where $\alpha$ is a scale parameter and $\theta$ is a shape parameter.

The survival (sf), hazard rate (hrf), and reversed hrf and cumulative hrf functions of TIITLIE model are

$$
\begin{array}{ll}
R(x ; \varphi)=\left[1-e^{-\frac{2 \alpha}{x}}\right]^{\theta}, & h(x ; \varphi)=\frac{2 \theta \alpha x^{-2} e^{-\frac{2 \alpha}{x}}}{1-e^{-\frac{2 \alpha}{x}}}, \\
\tau(x ; \varphi)=\frac{2 \theta \alpha x^{-2} e^{-\frac{2 \alpha}{x}}\left[1-e^{-\frac{2 \alpha}{x}}\right]^{\theta-1}}{1-\left[1-e^{-\frac{2 \alpha}{x}}\right]^{\theta}}, & H(x ; \varphi)=-\theta \ln \left[1-e^{-\frac{2 \alpha}{x}} .\right.
\end{array}
$$

Figure 1 shows some graphs of the pdf and hrf for TIITLIE with various values of parameters.
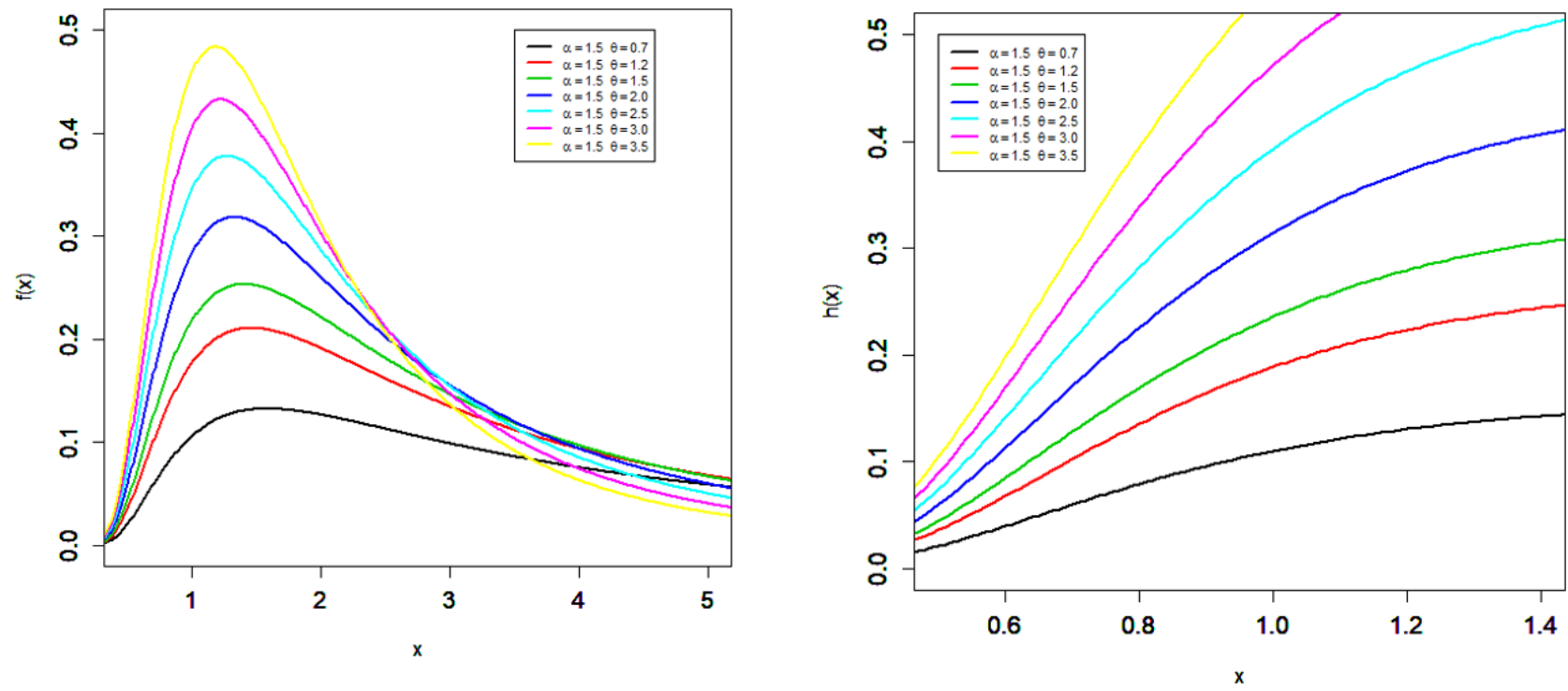

Figure 1: Plots of the pdf and hrf of the TIITLIE model. 
From Figure 1, we notice that pdf of TIITLIE model can be right skewed. Also the hrf can be increasing.

\section{Fundamental properties}

Some fundamental properties of the TIITLIE model are calculated in this section.

\subsection{Useful representation}

The two useful expansions of pdf and cdf for TIITLIE distribution is derived in this subsection. Using the next binomial expansions

$$
(1-a)^{n}=\sum_{i=0}^{\infty}(-1)^{i}\left(\begin{array}{c}
a \\
i
\end{array}\right) a^{i} .
$$

By using (3.1), the following term in (2.2), we get

$$
\left(1-e^{-\frac{2 \alpha}{x}}\right)^{\theta-1}=\sum_{i=0}^{\infty}(-1)^{i}\left(\begin{array}{c}
\theta-1 \\
i
\end{array}\right) e^{-\frac{2 \alpha}{\alpha}} .
$$

Inserting (3.2) in (2.2), then, the pdf of TIITLIE is

$$
f(x)=\alpha \sum_{i=0}^{\infty} W_{i} x^{-2} e^{-2(i+1) \frac{\alpha}{x}},
$$

where $w_{i}=2(-1)^{i}\left(\begin{array}{c}\theta-1 \\ i\end{array}\right) \theta$.

To get the expansion of $[F(x)]^{h}$, where $h$ is an integer,

$$
[\mathrm{F}(\mathrm{x})]^{\mathrm{h}}=\left[1-\left(1-e^{-2 \frac{\alpha}{x}}\right)^{\theta}\right]^{\mathrm{h}} .
$$

Then,

$$
[F(x)]^{h}=\sum_{k=0}^{\infty} W_{k} e^{-2 k \frac{\alpha}{x}}
$$

where, $w_{k}=\sum_{j=0}^{h}(-1)^{j+k}\left(\begin{array}{c}h \\ j\end{array}\right)\left(\begin{array}{c}\theta j \\ k\end{array}\right)$.

\subsection{Quantile function}

If $X$ has the $\operatorname{cdf}(2.1)$, then the quantile function is

$$
\mathrm{Q}(\mathrm{u})=\alpha\left(-\ln \sqrt{1-(1-\mathfrak{u})^{\frac{1}{\theta}}}\right)^{-1}, 0<\mathfrak{u}<1 .
$$

The median $(M)$ is given by

$$
M=\alpha\left(-\ln \sqrt{1-(0.5)^{\frac{1}{\theta}}}\right)^{-1} .
$$




\subsection{Moments}

If $X \sim$ TIITLIE model then, the $r^{\text {th }}$ moment is

$$
\mu_{r}^{\prime}=E\left(X^{r}\right)=\int_{-\infty}^{\infty} x^{r} f(x ; \varphi) d x
$$

Inserting (3.3) in (3.5), then

$$
\mu_{r}^{\backslash}=\alpha \sum_{i=0}^{\infty} W_{i} \int_{0}^{\infty} x^{r-2} e^{-2(i+1) \frac{\alpha}{x_{i}}} d x
$$

Let $y=\frac{\alpha}{x}$, then

$$
\mu_{\mathrm{r}} \backslash=\alpha^{r} \sum_{i=0}^{\infty} W_{i} \int_{0}^{\infty} y e^{-2(i+1) y} d y
$$

Then, $\mu_{\mathrm{r}}^{\prime}$ becomes

$$
\mu_{r} \backslash=\alpha^{r} \sum_{i=0}^{\infty} W_{i} \frac{\Gamma(1-r)}{(i+1)^{1-r}}, r<1
$$

The moment generating function of $X$ is

$$
M_{X}(t)=\sum_{r=0}^{\infty} \frac{t^{r}}{r !} E\left(X^{r}\right)=\sum_{r, i=0}^{\infty} \frac{t^{r}}{r !} \frac{w_{i} \Gamma(1-r)}{[i+1]^{1-r}}, r<1
$$

\subsection{Probability weighted moments}

The PMWs is given in the next equation

$$
\tau_{r, s}=E\left[X^{r} F(x)^{s}\right]=\int_{-\infty}^{\infty} x^{r} f(x)(F(x))^{s} d x .
$$

By inserting (3.3) and (3.4) into (3.6), replacing $h$ with $s$, leads to:

$$
\tau_{r, s}=\alpha \sum_{i, k=0}^{\infty} W_{i} W_{k} \int_{0}^{\infty} x^{r-2} e^{-2(i+k+1) \frac{\alpha}{x}} d x .
$$

Then,

$$
\tau_{r, s}=\alpha^{r} \sum_{i, k=0}^{\infty} W_{i} W_{k} \frac{\Gamma(1-r)}{(i+k+1)^{1-r}}, \quad r<1 .
$$

\subsection{Rényi entropy}

The Rényi entropy is defined by

$$
I_{\delta}(X)=\frac{1}{1-\delta} \log \int_{-\infty}^{\infty} f(x ; \varphi)^{\delta} d x, \delta>0 \text { and } \delta \neq 1
$$

By applying the binomial theory (3.1) in the pdf (2.2), then the $f(x ; \varphi)^{\delta}$ can be written as

$$
f(x ; \varphi)^{\delta}=\sum_{i=0}^{\infty} W_{i} x^{-2 \delta} e^{-\frac{2 \alpha[i+\delta]}{x}},
$$

where

$$
W_{i}=(2 \theta \alpha)^{\delta}(-1)^{i}\left(\begin{array}{c}
\delta(\theta-1) \\
i
\end{array}\right)
$$


Therefore, the Rényi entropy of TIITLIE distribution is

$$
\mathrm{I}_{\delta}(\mathrm{X})=\frac{1}{1-\delta} \log \left[\sum_{i=0}^{\infty} \mathrm{W}_{\mathrm{i}} \int_{0}^{\infty} x^{-2 \delta} e^{-\frac{2 \alpha[i+\delta]}{x}} \mathrm{~d} x\right],
$$

then,

$$
\mathrm{I}_{\delta}(\mathrm{X})=\frac{1}{1-\delta} \log \left[\sum_{i=0}^{\infty} \frac{\mathrm{W}_{\mathrm{i}} \Gamma(1-2 \delta)}{[2 \alpha[i+\delta]]^{2 \delta-1}}\right]
$$

\section{ML estimation}

Let $X_{1}, \ldots, X_{n}$ be observed values from the TIITLIE model with parameters $\varphi=(\alpha, \theta)^{\top}$. The total log-likelihood function is

$$
\ln L(\varphi)=n \ln 2 \theta+n \ln \alpha-2 \sum_{i=1}^{n} \ln x_{i}-2 \sum_{i=1}^{n}\left(\frac{\alpha}{x_{i}}\right)+(\theta-1) \sum_{i=1}^{n} \ln \left[1-e^{-2\left(\frac{\alpha}{x_{i}}\right)}\right] .
$$

The elements of the score function $\mathrm{U}(\varphi)=\left(\mathrm{U}_{\alpha}, \mathrm{U}_{\theta}\right)$ are

$$
\mathrm{u}_{\alpha}=\frac{n}{\alpha}-\sum_{i=1}^{n} x_{i}^{-1}+2(\theta-1) \sum_{i=1}^{n} \frac{x_{i}^{-1} e^{-2\left(\frac{\alpha}{x_{i}}\right)}}{1-e^{-2\left(\frac{\alpha}{x_{i}}\right)}}, \quad \text { and } \quad u_{\theta}=\frac{n}{\theta}+\sum_{i=1}^{n} \ln \left[1-e^{-2\left(\frac{\alpha}{x_{i}}\right)}\right] \text {. }
$$

Then the ML estimators of the parameters $\varphi$ are investigated by putting $\mathrm{U}_{\varphi}$ to be zero and solving them. The numerical results is showed in Tables 1-3.

Table 1: The parameter estimation from TIITLIE model.

\begin{tabular}{|c|c|c|c|c|}
\hline \multirow{2}{*}{$\mathrm{n}$} & \multicolumn{2}{|c|}{$\alpha=0.5, \theta=0.5$} & \multicolumn{2}{c|}{$\alpha=1.5, \theta=0.5$} \\
\cline { 2 - 5 } & MLE & MSE & MLE & MSE \\
\hline \multirow{2}{*}{50} & 0.519 & 0.010 & 0.651 & 0.574 \\
\cline { 2 - 5 } & 0.529 & 0.018 & 1.591 & 0.176 \\
\hline \multirow{2}{*}{100} & 0.511 & 0.006 & 0.559 & 0.077 \\
\cline { 2 - 5 } & 0.516 & 0.010 & 1.546 & 0.095 \\
\hline \multirow{2}{*}{200} & 0.506 & 0.003 & 0.530 & 0.020 \\
\cline { 2 - 5 } & 0.509 & 0.005 & 1.530 & 0.044 \\
\hline \multirow{2}{*}{500} & 0.502 & 0.001 & 0.508 & 0.005 \\
\cline { 2 - 5 } & 0.503 & 0.002 & 1.508 & 0.014 \\
\hline
\end{tabular}

Table 2: The parameter estimation from TIITLIE model.

\begin{tabular}{|c|c|c|c|c|}
\hline \multirow{2}{*}{$\mathrm{n}$} & \multicolumn{2}{|c|}{$\alpha=1.5, \theta=1.5$} & \multicolumn{2}{c|}{$\alpha=0.5, \theta=1$} \\
\cline { 2 - 5 } & MLE & MSE & MLE & MSE \\
\hline \multirow{2}{*}{50} & 1.898 & 5.603 & 1.035 & 0.037 \\
\cline { 2 - 5 } & 1.592 & 0.171 & 0.531 & 0.020 \\
\hline \multirow{2}{*}{100} & 1.709 & 0.637 & 1.021 & 0.024 \\
\cline { 2 - 5 } & 1.550 & 0.097 & 0.516 & 0.011 \\
\hline 200 & 1.585 & 0.164 & 1.011 & 0.012 \\
\cline { 2 - 5 } & 1.526 & 0.043 & 0.508 & 0.005 \\
\hline 500 & 1.522 & 0.043 & 1.004 & 0.004 \\
\cline { 2 - 5 } & 1.507 & 0.014 & 0.504 & 0.002 \\
\hline
\end{tabular}


Table 3: The parameter estimation from TIITLIE model.

\begin{tabular}{|c|c|c|c|c|}
\hline \multirow{2}{*}{$\mathrm{n}$} & \multicolumn{2}{|c|}{$\alpha=0.5, \theta=1.5$} & \multicolumn{2}{c|}{$\alpha=1, \theta=0.5$} \\
\cline { 2 - 5 } & MLE & MLE & MLE & MSE \\
\hline \multirow{2}{*}{50} & 1.561 & 0.552 & 0.552 & 0.036 \\
\cline { 2 - 5 } & 0.535 & 1.066 & 1.066 & 0.083 \\
\hline \multirow{2}{*}{100} & 1.536 & 0.529 & 0.529 & 0.017 \\
\cline { 2 - 5 } & 0.520 & 1.039 & 1.039 & 0.041 \\
\hline 200 & 1.512 & 0.512 & 0.512 & 0.008 \\
\cline { 2 - 5 } & 0.508 & 1.018 & 1.018 & 0.020 \\
\hline 500 & 1.506 & 0.506 & 0.506 & 0.002 \\
\cline { 2 - 5 } & 0.504 & 1.007 & 1.007 & 0.006 \\
\hline
\end{tabular}

\section{Application}

In this section, one application to a real data set is performed to show the superiority of the TIITLIE model against IE model. The data set is taken from Tahir et al. [8] represents Failure times of 63 aircraft windshields.

The MLEs, standard errors (SEs) of the TIITLIE parameters, the minus two log-likelihood $(-2 \log \mathrm{L})$ and Kolmogorov-smirnov (k-s) are mentioned in Table 4.

Table 4: MLEs and SEs, $-2 \log \mathrm{L}$ and $\mathrm{k}$-s for the data set.

\begin{tabular}{|l|c|c|c|c|}
\hline Model & \multicolumn{2}{|c|}{ MLE and SE } & $-2 \log \mathrm{L}$ & k-s \\
\hline $\operatorname{TIITLIE}(\alpha, \theta)$ & $\begin{array}{c}0.365 \\
(0.067)\end{array}$ & $\begin{array}{c}0.912 \\
(0.161)\end{array}$ & 270.299 & 0.27504 \\
\hline $\operatorname{IE}(\alpha)$ & $\begin{array}{c}0.782 \\
(0.002)\end{array}$ & - & 270.575 & 0.28158 \\
\hline
\end{tabular}

Table 4 compares the fits of the TIITLIE model against the IE model. Also, show the superiority of the TIITLIE model against IE model. The estimated cdf and estimated sf plots for the competitive models are mentioned in Figures 2 and 3.

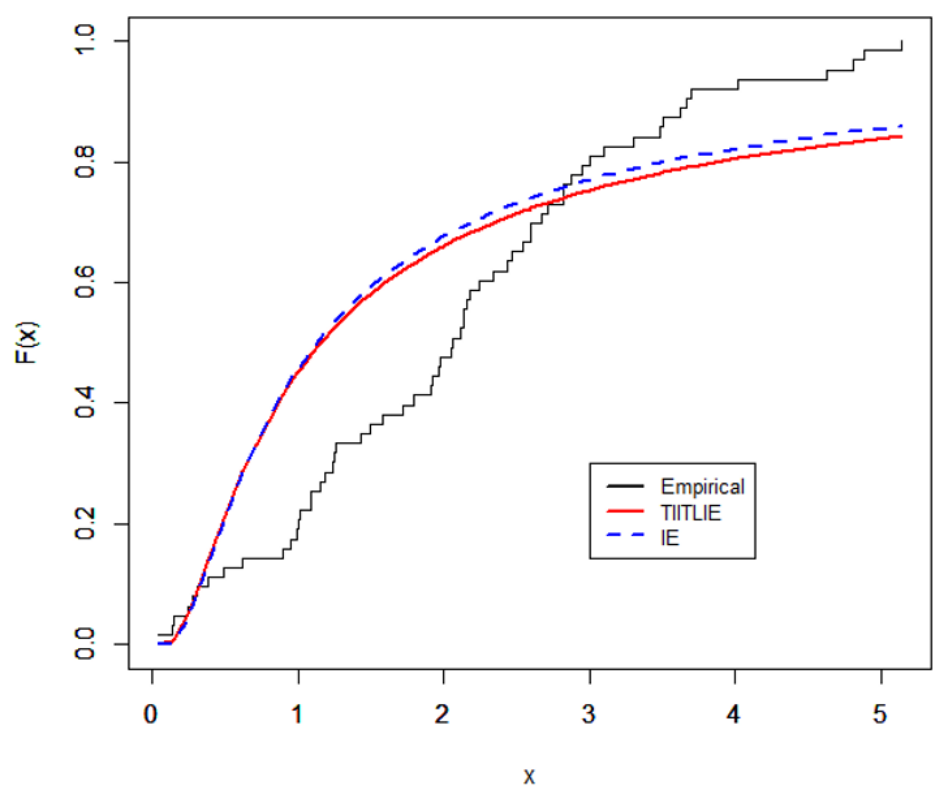

Figure 2: The estimated cdf of the fitted models. 


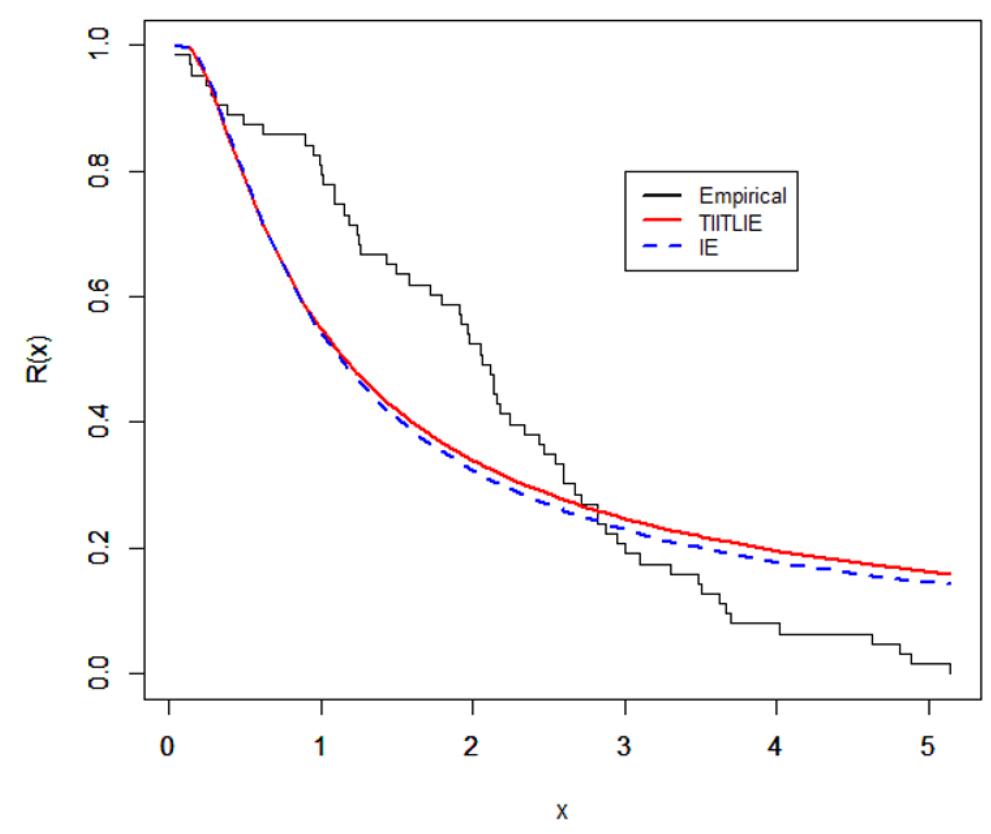

Figure 3: The estimated sf of the fitted models.

\section{Conclusions}

In this article, we introduce and study a new lifetime model, which called the TIITLIE model. We investigate some of its fundamental properties of the TIITLIE model. ML method of estimation is assessed to the parameters of the new model. The superiority of the TIITLIE model against IE model is showed by using the 63 aircraft windshields real data set.

\section{References}

[1] M. Elgarhy, M. Arslan Nasir, F. Jamal, G. Ozel, The type II topp-leone generated family of distributions: Properties and applications, J. Stat. Manag. Syst., 21 (2018), 1529-1551. 1

[2] A. Z. Keller, A. R. Kamath, Reliability analysis of CNC Machine Tools, Reliab. Eng., 3 (1982), 449-473. 1

[3] P. E. Oguntunde, A. O. Adejumo, E. A. Owoloko, Application of Kumaraswamy inverse exponential distribution to real lifetime data, Int. J. Appl. Math. Stat., 56 (2017), 34-47. 1

[4] P. E. Oguntunde, A. O. Adejumo, E. A. Owoloko, On the flexibility of the transmuted inverse exponential distribution, Lecture Notes on Engineering and Computer Science: Proceeding of the World Congress on Engineering (London, U.K.), 2017 (2017), 123-126. 1

[5] P. E. Oguntunde, A. O. Adejumo, E. A. Owoloko, On the exponentiated generalized inverse exponential distribution, Lecture Notes on Engineering and Computer Science: Proceeding of the World Congress on Engineering (London, U.K.), 2017 (2017), 80-83. 1

[6] P. E. Oguntunde, A. O. Adejumo, E. A. Owoloko, The Weibull-inverted exponential distribution: A generalization of the inverse exponential distribution, Lecture Notes on Engineering and Computer Science: Proceeding of the World Congress on Engineering (London, U.K.), 2017 (2017), 16-19. 1

[7] B. Singh, R. Goel, The beta inverted exponential distribution: Properties and applications, Int. J. Appl. Sci. Math., 2 (2015), 132-141. 1

[8] M. H. Tahir, G. M. Cordeiro, M. Mansoor, M. Zubair, The Weibull-Lomax distribution: properties and applications, Hacet. J. Math. Stat., 44 (2015), 455-474. 5 